\title{
A systematic review of serum autoantibodies as biomarkers for pancreatic cancer detection
}

\author{
Karin Dumstrei ${ }^{1,2}$, Hongda Chen ${ }^{1}$, Hermann Brenner ${ }^{1,3,4}$ \\ ${ }^{1}$ Division of Clinical Epidemiology and Aging Research, German Cancer Research Center (DKFZ), D-69120 Heidelberg, \\ Germany \\ ${ }^{2}$ European Molecular Biology Organization (EMBO), D-69117 Heidelberg, Germany \\ ${ }^{3}$ Division of Preventive Oncology, German Cancer Research Center (DKFZ) and National Center for Tumor Diseases (NCT), \\ D-69120 Heidelberg, Germany \\ ${ }^{4}$ German Cancer Consortium (DKTK), German Cancer Research Center (DKFZ), D-69120 Heidelberg, Germany
}

Correspondence to: Hermann Brenner, e-mail: h.brenner@dkfz.de

Keywords: autoantibodies, biomarkers, pancreatic cancer, early detection, systematic review

Received: September 28, 2015

Accepted: January 18, 2016

Published: January 31, 2016

\section{ABSTRACT}

Pancreatic cancer is a leading cause of cancer-related deaths in the western world. Patients with pancreatic cancer have poor prognosis, partly due to difficulties in detecting it at early stages. While different markers have been associated with pancreatic cancer, many of them show suboptimal sensitivity and specificity. Serum autoantibodies against tumor-associated antigens have recently emerged as early stage biomarkers for different types of cancers. Given the urgent need for early and reliable biomarkers for pancreatic cancer, we undertook a systematic review of the published literature to identify primary articles that evaluated serum autoantibodies in pancreatic cancer detection by searching PubMed and ISI Web of Knowledge. Two reviewers extracted data on study characteristics and results independently. Overall, 31 studies evaluating 124 individual serum autoantibodies in pancreatic cancer detection met the inclusion criteria. In general, single autoantibody markers showed relatively low sensitivities at high specificity. A combination of markers, either multiple serum autoantibodies or serum autoantibodies combined with tumorassociated markers, led to a better diagnostic performance. However, most of the analyzed autoantibodies have only been reported in single studies and therefore need to be independently validated. We conclude that serum autoantibodies might present an option as biomarkers for early detection of pancreatic cancer, but more work is needed to identify and validate autoantibody signatures that are associated with early stage pancreatic cancer.

\section{INTRODUCTION}

Pancreatic cancer is one of the most common causes of cancer related deaths and represents a serious health problem. In the US, pancreatic cancer is the 4th leading cause of cancer death [1]. The vast majority of cases are seen in patients above the age of 55 with the median age of onset being 71 [2]. What makes the outlook for pancreatic cancer particularly troubling is the poor prognosis. The five-year relative survival rates are commonly below $10 \%$ and the incidence rate matches closely to the mortality rate. In the US, 46420 new cases were expected in 2014 while 39590 deaths were anticipated. One of the reasons for the poor prognosis is that most patients have locally advanced or metastatic cancer at time of diagnosis. $53 \%$ of patients are diagnosed at late stages with a 5 -year survival rate of $2 \%$, but even for the $9 \%$ percent of patients that are diagnosed with local cancer the 5-year relative survival rate is only $24 \%$ [1].

Most cases of pancreatic cancer are sporadic with the major risk factors being aging, smoking, diabetes, chronic pancreatitis and obesity $[2,3]$. Inherited genetic factors are thought to contribute to $5-10 \%$ of pancreatic cancers [3-5]. Mutations in a number of different genes like, the familial breast cancer associated gene BRAC2 and its binding partner PALB2 lead to increased risk of 
developing pancreatic cancer. Some studies have also linked BRAC1 to pancreatic cancer, but the evidence is less strong as compared to BRAC2. Mutations in the cell cycle regulator $p 16 / \mathrm{CDKN} 2 \mathrm{~A}$ are also associated with familiar pancreatic cancer as are mutations in the serine/ threonine kinase STK11/LKB1 [3-5].

Given the relative low incidence rate of pancreatic cancer, screening of the general population is in general not recommended. However, experts do agree on the benefit of screening patients that are at high risk for developing pancreatic cancer [6]. As there are currently no reliable biomarkers or screening tools available for detecting early stages of pancreatic cancer there is, however, no consensus for the most effective screening protocol

There have been many tumor-associated markers described for pancreatic cancer. Some of the bettercharacterized ones are CA19-9, CA-50 and CEA (reviewed in [7]). However, these markers tend to show suboptimal sensitivity and specificity [7]. CA19-9, for example, is also overexpressed in other types of gastrointestinal cancers and in inflammatory conditions such as pancreatitis and is thus not specific for pancreatic cancer [7]. There is therefore an urgent need to find better biomarkers and more accurate diagnostic tools.

The immune system also reacts to developing tumors and generates autoantibodies against tumorassociated antigens (TAA). This has led to a search for serological autoantibodies and their respective antigens in different types of cancers $[8,9]$. While the mechanism of autoantibody production is not fully clear, cancer patients do produce autoantibodies to proteins that are either mutated, misfolded, overexpressed or to proteins that show altered post-translational modifications like glycosylation. Recent work supports that serum autoantibodies may be suitable biomarkers that can be used either alone or in combination with tumor associated markers or other autoantibodies for detection of cancers $[8,9]$. The hope is that one can come up with defined autoantibody signatures for different types of cancers and tumor stages that can be used to detect cancers at early stages.

A number of studies have evaluated serological autoantibodies in pancreatic cancer. However, so far no comprehensive review of these studies has been done. We provide here a systematic review of the published literature to identify articles that have looked at serum autoantibodies in pancreatic cancer. We report the key aspects of the study design and population characteristics, the sensitivity and specificity of the investigated autoantibodies and marker combinations performed to provide a review of where the field stands at this stage.

\section{RESULTS}

The literature search process is shown in Figure 1. Overall, we identified 1836 articles using PubMed and Web of Science searches. Of these 189 were duplicates, 131 non-English and 138 were reviews/abstracts. Based upon

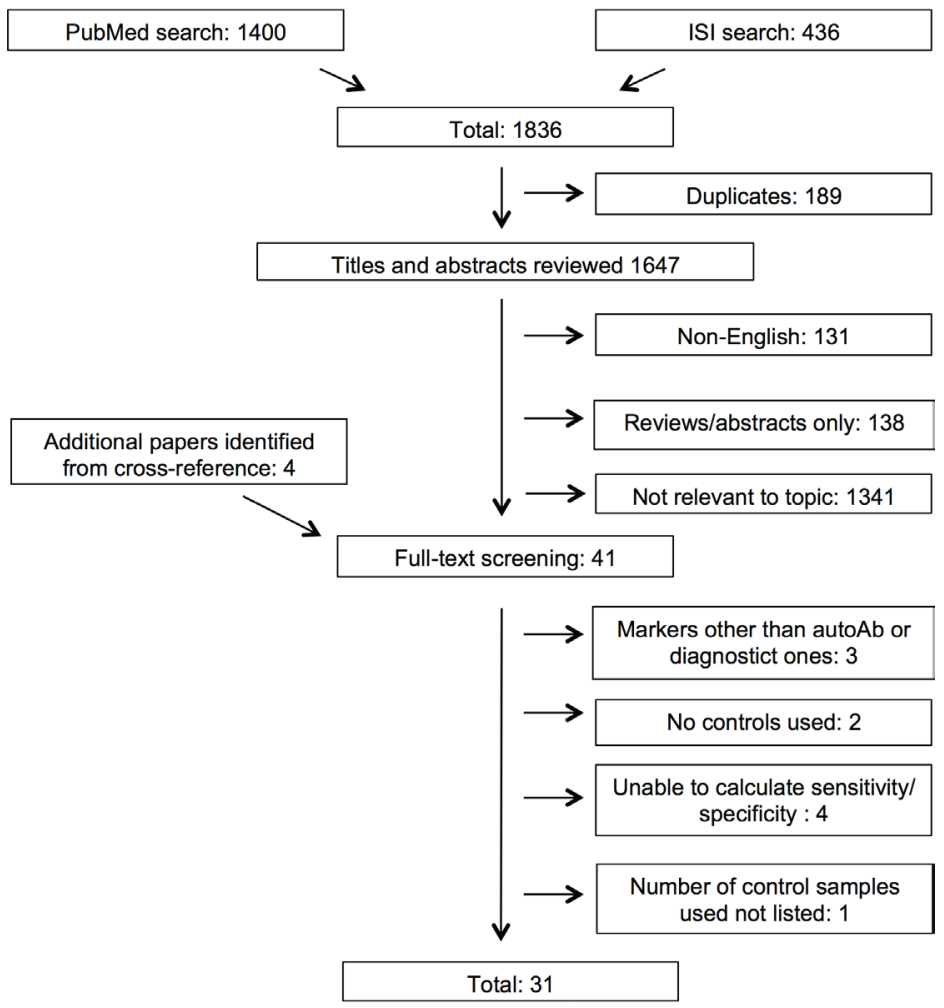

Figure 1: Overview of the literature search process (until 27th of April 2015). 
title/abstract reading 1341 articles were not relevant to the topic leading to the full text screening of 41 articles. Ten of these were excluded due to the following reasons (see also Supplementary File S1): three studies evaluated markers other than autoantibodies or diagnostic ones [10-12], two studies lacked cancer free controls $[13,14]$, we were unable to calculate sensitivity and specificity in four studies [15-18] and one study did not provide the number of included controls [19] In the end, with four articles additionally identified through cross-referencing [20-23], 31 articles were included in our review [20-50].

\section{Study characteristics}

The key study characteristics are provided in Supplementary Table S2. This table shows for each study the number of cases and controls, the age range and average age, male/female ratio, the status of the controls as well as the detection method used to evaluate serum autoantibodies. 10 studies [20, 23-25, 28, 29, 42, $44,47,48]$ provided complete information on all these variables. The median numbers of pancreatic cases and corresponding controls included were 47 (range: 8-300) and 43 (range: 5-436), respectively.

Healthy controls were used in 25 studies, while in the other studies a mixture of patients with noncancer pancreatic diseases (e.g., chronic pancreatitis) were used. One study used a random random-digit dial method to select controls and didn't specify the status of the controls in terms of non-cancer pancreatic diseases or other types of cancers [24]. Tumor stage information was provided in two studies [20,35]. The age range and average age were reported in 10 studies, while in 14 studies the average age was provided. In studies where the age information was provided, most of them showed a fairly similar age distribution among cases and controls.

Different techniques were used to detect serum autoantibodies with the most common one being ELISA (17 studies). Western blot analysis and different proteomic approaches were also used in some studies. Within the last five years the use of proteomic analysis has become a more frequent choice for this type of analysis. In most studies, recombinant full-length proteins were used as antigen for autoantibodies. However in some studies, peptides [39, 45, 46] and glycosylated proteins [25] also served as antigens.

\section{Diagnostic performance of autoantibodies}

Overall, 124 individual autoantibodies were evaluated in the 31 included studies. The diagnostic performance of these autoantibodies, ordered by reported sensitivity, is listed in Table 1. The diagnostic performance of the autoantibodies varied greatly in terms of sensitivity and specificity. The sensitivity ranged from $0 \%$ to $100 \%$ with a median of $14 \%$ (average is $22 \%$ ). In general, the majority of markers showed a relative low sensitivity. 105 of the examined autoantibodies $(85 \%)$ showed less than $50 \%$ sensitivity. The specificity ranged from $55 \%$ to $100 \%$ with a median of $100 \%$ (average $95 \%$ ) and $85 \%$ of autoantibodies showed specificity greater or equal to $90 \%$. Four autoantibodies showed high specificity $(>80 \%)$ along with high sensitivity $(>60 \%)$. These markers are anti-Coactosin-like protein (CLP) peptide 104-113 [39], anti-Mesothelin [32], anti-Ezrin [26] and anti-ENOA1,2 [48] - see also Figure 2. However, it is important to note that the diagnostic performance of these autoantibodies has not been validated in other independent studies and case numbers in some of the studies were very small. AUC values were reported for 15 autoantibodies, but no internal or external validations were applied to adjust for potential overoptimism. Figure 2 shows a graphical representation of the sensitivities and specificities for all examined autoantibodies. As can be seen from Figure 2 as well as from Table 1, autoantibodies that showed high sensitivity tended to show lower specificity. Conversely, markers with low sensitivity tended to have high specificity.

Three autoantibodies, anti-p53, anti-PGK1 and anti-Annexin A2, were examined in multiple studies (Table 2). Given the prominent role of p53 in multiple cancers it is perhaps not surprising that this was the most commonly assessed autoantibody. Autoantibodies against p53 were evaluated in 8 studies [20, 34-37, 40, 41, 43]. As can be seen from Table 2, the sensitivities reported for autoantibodies against p53 varied between studies (6-28\%) while there was less variance in the specificity (range 85-90\%). PGK1 [21, 42] and Annexin A2 [21, 26] were each evaluated in two studies and both show relative low sensitivity, but high specificity.

Several studies also reported the sensitivity and specificity of a combination of different markers (Table 3 ). These studies looked at either multiple autoantibodies or autoantibodies combined with other tumor associated markers. The combination of markers generally led to enhanced sensitivity while maintaining relatively high specificity. For example the combination of anti-Ezrin and anti-ENOA1.2 with the tumor associated marker CA19.9 [26] lead to a sensitivity of $100 \%$. Again, as observed with individual autoantibodies, autoantibodies against p53 were a common choice for the maker combinations. The two most commonly used markers were anti-p53 and CA19.9, which were included in 11 and 8 of the 31 possible multimarker combinations, respectively. What is also evident from this analysis is the variability between different studies. Three studies looked at anti-p53 in combination with CA19.9 [37, 40, 43] and the reported sensitivity ranged from $26 \%$ to $73 \%$, while the specificity varied from $64 \%$ to $100 \%$.

Two studies also reported sensitivity according to tumor stage $[20,35]$ (Supplementary Table S3) for 6 different autoantibodies. From this analysis, one can see that the diagnostic performance of the autoantibodies was mostly higher in more advanced tumor stages. The sensitivity for autoantibodies at tumor stages I and II was in the range from $3 \%$ to $13 \%$ with the exception of anti-p16 that showed a sensitivity of $33 \%$ at tumor stage I. 
Table 1: Diagnostic performance of antibodies markers ordered by reported sensitivity

\begin{tabular}{|c|c|c|c|c|c|c|}
\hline First author, Year [Ref] & Antigen & $\begin{array}{c}\text { Cases }(\mathbf{N}) / \\
\text { Controls } \\
(\mathbf{N})\end{array}$ & $\begin{array}{l}\text { Sensitivity } \\
\%(95 \% \text { CI) }\end{array}$ & $\begin{array}{c}\text { Specificity \% } \\
\text { (95\% CI) }\end{array}$ & AUC & $p$-value* \\
\hline Nakatsura, 2002 [39] & CLP peptide 104-113 IgG & $8 / 9$ & $100(63-100)$ & $100(66-100)$ & - & - \\
\hline Johnston, 2009 [32] & Mesothelin & $74 / 5$ & $99(93-100)$ & $100(48-100)$ & - & $p<0.05$ \\
\hline Capello, 2013 [26] & Ezrin & $69 / 94$ & $93(83-98)$ & $76(66-84)$ & 0.9 & $p<0.0001$ \\
\hline Nagayoshi, 2014 [38] & TNP1 & $37 / 20$ & $89(75-97)$ & $55(32-77)$ & 0.732 & - \\
\hline Nagayoshi, 2014 [38] & CIB1 & $37 / 20$ & $76(59-88)$ & $70(46-88)$ & 0.753 & - \\
\hline Nagayoshi, 2014 [38] & RIT2 & $37 / 20$ & $76(59-88)$ & $65(41-85)$ & 0.704 & - \\
\hline Falco, 2013 [27] & Bag3 & $52 / 44$ & $75(61-86)$ & $76(60-87)$ & 0.77 & $\begin{array}{c}p= \\
0.00001\end{array}$ \\
\hline Nagayoshi, 2014 [38] & GABARAPL2 & $37 / 20$ & $68(50-82)$ & $75(51-91)$ & 0.674 & - \\
\hline Nagayoshi, 2014 [38] & KIAA0409 & $37 / 20$ & $65(47-80)$ & $70(46-88)$ & 0.72 & - \\
\hline Nakatsura, 2002 [39] & CLP peptide 15-24 IgE & $8 / 9$ & $63(24-91)$ & $56(21-86)$ & - & - \\
\hline Nagayoshi, 2014 [38] & DTYMK & $37 / 20$ & $62(45-78)$ & $75(51-91)$ & 0.691 & - \\
\hline Nagayoshi, 2014 [38] & STK33 & $37 / 20$ & $62(45-78)$ & $75(51-91)$ & 0.668 & - \\
\hline Tomaino, 2011 [48] & ENOA1,2 & $61 / 63$ & $62(49-74)$ & $100(94-100)$ & - & $p=0.0001$ \\
\hline Capello, 2013 [26] & Ezrin & $120 / 40$ & $56(46-65)$ & $90(76-97)$ & - & $p<0.0001$ \\
\hline Tanaka, 2007 [45] & PSCA peptide 2-11 & $40 / 60$ & $55(38-71)$ & $90(79-96)$ & - & $p=0.0001$ \\
\hline Nagayoshi, 2014 [38] & PCNA & $37 / 20$ & $54(37-71)$ & $85(62-97)$ & 0.669 & - \\
\hline Capello, 2013 [26] & Annexin A2 & $120 / 40$ & $53(44-62)$ & $90(76-97)$ & - & $p<0.0001$ \\
\hline Tanaka, 2007 [45] & PSCA peptide $86-95$ & $40 / 60$ & $53(36-68)$ & $90(79-96)$ & - & $p=0.0001$ \\
\hline Kamei, 1992 [33] & Histone H2B & $8 / 45$ & $50(16-84)$ & $93(82-99)$ & - & - \\
\hline Nagayoshi, 2014 [38] & EIF3S4 & $37 / 20$ & $49(32-66)$ & 85 (62-97) & 0.67 & - \\
\hline Syrigos, 1996 [44] & Insulin & $36 / 21$ & $48(30-65)$ & $100(84-100)$ & - & - \\
\hline Hong, 2004 [31] & Calreticulin isoform 2 & $36 / 15$ & $44(28-62)$ & $100(78-100)$ & - & - \\
\hline Tanaka, 2006 [46] & SART-109 peptide & $47 / 42$ & $43(28-58)$ & $79(63-90)$ & - & $p<0.05$ \\
\hline Hong, 2004 [31] & Calreticulin isoform 1 & $36 / 15$ & $42(26-60)$ & $93(68-100)$ & - & - \\
\hline Capello, 2013 [26] & Ezrin & $16 / 32$ & $38(15-65)$ & $100(89-100)$ & - & $p=0.0002$ \\
\hline Nakatsura, 2002 [39] & CLP peptide 104-113 IgE & $8 / 9$ & $37(9-76)$ & $78(40-97)$ & - & - \\
\hline Capello, 2013 [26] & hnRNPL & $120 / 40$ & $35(27-44)$ & $95(83-99)$ & - & $p<0.001$ \\
\hline Xia, 2005 [49] & \begin{tabular}{|l|} 
DDX48 \\
\end{tabular} & $60 / 60$ & $33(22-47)$ & $100(94-100)$ & - & $p<0.01$ \\
\hline Tanaka, 2006 [46] & EGFR-479 peptide & $47 / 42$ & $32(19-47)$ & $91(77-97)$ & - & $p<0.05$ \\
\hline Capello, 2013 [26] & Vinculin & $120 / 40$ & $31(23-40)$ & $95(83-99)$ & - & $p=0005$ \\
\hline Pekarikova, 2010 [23] & Calreticulin IgG & $55 / 56$ & $31(19-45)$ & $98(90-100)$ & - & - \\
\hline Li, $2012[35]$ & p16 & $23 / 23$ & $30(13-53)$ & $96(78-100)$ & - & $p<0.05$ \\
\hline Tanaka, 2007 [45] & PSCA peptide 109-118 & $40 / 60$ & $30(17-47)$ & $95(86-99)$ & - & - \\
\hline Laurent-Puig, 1995 [34] & $\mathrm{p} 53$ & $29 / 33$ & $28(13-47)$ & $85(68-95)$ & - & - \\
\hline Tanaka, 2007 [45] & PSCA peptide 108-117 & $40 / 60$ & $28(15-44)$ & $93(84-98)$ & - & $p=0.0431$ \\
\hline Tomaino, 2007 [47] & TAGL or COF1 & $70 / 40$ & $27(17-39)$ & $100(91-100)$ & - & $p=0.002$ \\
\hline $\mathrm{Li}, 2012[35]$ & IMP1 & $23 / 23$ & $26(10-48)$ & $96(78-100)$ & - & - \\
\hline Patwa, 2009 [42] & Histone H4 & $54 / 94$ & $54(15-40)$ & $96(89-99)$ & - & - \\
\hline Pekarikova, 2010 [23] & Calreticulin IgA & $55 / 56$ & $25(15-39)$ & $100(94-100)$ & - & - \\
\hline Ohshio, $2002[40]$ & p53 & $82 / 21$ & $23(15-34)$ & $95(76-100)$ & - & - \\
\hline
\end{tabular}




\begin{tabular}{|c|c|c|c|c|c|c|}
\hline Tomaino, 2007 [47] & TPIS & $70 / 40$ & $23(14-34)$ & $100(91-100)$ & - & $p=0.004$ \\
\hline Muller, 2006 [37] & $\mathrm{p} 53$ & $22 / 436$ & $23(8-45)$ & $100(99-100)$ & - & - \\
\hline Tanaka, 2007 [45] & PSCA peptide 18-27 & $40 / 60$ & $23(11-38)$ & $93(84-98)$ & - & $p=0.0105$ \\
\hline Li, $2012[35]$ & p62 & $23 / 23$ & $22(7-44)$ & $100(85-100)$ & - & - \\
\hline Li, 2012 [35] & Koc & $23 / 23$ & $22(7-44)$ & $100(85-100)$ & - & - \\
\hline Okada, 2005 [41] & Kinectin1 & $37 / 34$ & $22(10-38)$ & $88(73-97)$ & - & - \\
\hline Capello, 2013 [26] & PDC6I & $120 / 40$ & $21(14-29)$ & $97(87-100)$ & - & $p=0.0033$ \\
\hline Tomaino, 2007 [47] & $\mathrm{K} 1 \mathrm{C} 10$ & $70 / 40$ & $21(13-33)$ & $100(91-100)$ & - & $p=0.005$ \\
\hline $\mathrm{Li}, 2010[21]$ & PGK1 & $48 / 40$ & $21(10-35)$ & $100(91-100)$ & - & - \\
\hline Tanaka, 2007 [45] & PSCA peptide 51-60 & $40 / 60$ & $20(9-36)$ & $93(84-98)$ & - & $p=0.0155$ \\
\hline Tanaka, 2007 [45] & PSCA peptide 27-37 & $40 / 60$ & $20(9-36)$ & $93(84-98)$ & - & - \\
\hline Tomaino, 2007 [47] & AL1A1 & $70 / 40$ & $20(11-31)$ & $100(91-100)$ & - & $p=0.006$ \\
\hline Tanaka, 2006 [46] & Pap-112 peptide & $47 / 42$ & $19(9-33)$ & $91(77-97)$ & - & $p>0.05$ \\
\hline Capello, 2013 [26] & Annexin A1 & $120 / 40$ & $19(13-27)$ & $100(91-100)$ & - & $p=0.0012$ \\
\hline Raedle, 1996 [43] & $\mathrm{p} 53$ & $33 / 52$ & $18(7-35)$ & $90(79-97)$ & - & - \\
\hline Tanaka, 2007 [45] & PSCA peptide $44-52$ & $40 / 60$ & $18(7-33)$ & $93(84-98)$ & - & $p=0.0398$ \\
\hline Li, $2012[35]$ & p53 & $23 / 23$ & $17(5-39)$ & $100(85-100)$ & - & - \\
\hline Li, $2012[35]$ & Survivin & $23 / 23$ & $17(5-39)$ & $96(78-100)$ & - & - \\
\hline Tanaka, 2006 [46] & EGFR-54 peptide & $47 / 42$ & $17(8-31)$ & $95(84-99)$ & - & $p>0.05$ \\
\hline Tanaka, 2006 [46] & CEA-425 peptide & $47 / 42$ & $17(8-31)$ & $93(81-99)$ & - & $p>0.05$ \\
\hline $\mathrm{Li}, 2010[21]$ & MDH1 & $48 / 40$ & $17(8-31)$ & $100(91-100)$ & - & - \\
\hline Gansange, 1996 [20] & $\mathrm{p} 53$ & $145 / 60$ & $16(10-23)$ & $100(94-100)$ & - & - \\
\hline Heller, $2010[30]$ & MIA & $34 / 20$ & $15(5-31)$ & $94(75-100)$ & - & - \\
\hline Tomaino, 2007 [47] & TPIS & $70 / 40$ & $14(7-25)$ & $100(91-100)$ & - & $p=0.004$ \\
\hline Okada, 2005 [41] & $\mathrm{hMSH} 2$ & $37 / 34$ & $14(5-29)$ & $100(90-100)$ & - & - \\
\hline Okada, 2005 [41] & IMAGE:3480396 3' & $37 / 34$ & $14(5-29)$ & $88(73-97)$ & - & - \\
\hline Gnjatic, 2010 [29] & NR2E3 & $60 / 53$ & $13(6-25)$ & $96(87-100)$ & - & - \\
\hline Tomaino, 2007 [47] & G6PD & $70 / 40$ & $13(6-23)$ & $100(91-100)$ & - & $p=0.03$ \\
\hline Tomaino, 2007 [47] & IDHC & $70 / 40$ & $13(6-23)$ & $100(91-100)$ & - & $p=0.03$ \\
\hline Patwa, 2009 [42] & PGK1 & $49 / 43$ & $12(5-25)$ & $97(88-100)$ & - & - \\
\hline Gnjatic, 2010[29] & ROR2 & $60 / 53$ & $12(5-23)$ & $96(87-100)$ & - & - \\
\hline Heller, $2010[30]$ & PNLIPRP2 & $34 / 20$ & $12(3-28)$ & $100(83-100)$ & - & - \\
\hline Tomaino, 2007 [47] & EFTU & $70 / 40$ & $11(5-21)$ & $100(91-100)$ & - & $p=0.04$ \\
\hline Okada, 2005 [41] & KIAA0580 & $37 / 34$ & $11(3-25)$ & $94(80-99)$ & - & - \\
\hline Okada, 2005 [41] & RUNX2 & $37 / 34$ & $1(3-25)$ & $88(73-97)$ & - & - \\
\hline Tanaka, 2007 [45] & PSCA peptide 3-11 & $40 / 60$ & $10(3-24)$ & $95(86-99)$ & - & $p=0.0000$ \\
\hline Tanaka, 2007 [45] & PSCA peptide 3-12 & $40 / 60$ & $10(3-24)$ & $96(88-100)$ & - & $p=0.0000$ \\
\hline Heller, 2010 [30] & IFITM3 & $34 / 20$ & $9(2-24)$ & $100(83-100)$ & - & - \\
\hline $\mathrm{Li}, 2010[21]$ & ARFIP2 & $48 / 40$ & $8(2-20)$ & $100(91-100)$ & - & - \\
\hline Okada, 2005 [41] & hPMS1 & $37 / 34$ & $8(2-22)$ & $100(90-100)$ & - & - \\
\hline Okada, 2005 [41] & HRY & $37 / 34$ & $8(2-22)$ & $100(90-100)$ & - & - \\
\hline Gnjatic, 2010 [29] & MAPK9 & $60 / 53$ & $8(3-18)$ & $100(93-100)$ & - & - \\
\hline Gnjatic, 2010 [29] & C6orf141 & $60 / 53$ & $8(3-18)$ & $100(93-100)$ & - & - \\
\hline Gnjatic, 2010 [29] & MAPK9 & $60 / 53$ & $8(3-18)$ & $100(93-100)$ & - & - \\
\hline Gnjatic, 2010 [29] & GAS2 & $60 / 53$ & $8(3-18)$ & $98(90-100)$ & - & - \\
\hline
\end{tabular}




\begin{tabular}{|c|c|c|c|c|c|c|}
\hline Gnjatic, 2010 [29] & KIAA1618 & $60 / 53$ & $8(3-18)$ & $98(90-100)$ & - & - \\
\hline Gnjatic, 2010 [29] & PTPRA & $60 / 53$ & $7(2-16)$ & $100(93-100)$ & - & - \\
\hline Gnjatic, 2010 [29] & LRRC49 & $60 / 53$ & $7(2-16)$ & $100(93-100)$ & - & - \\
\hline Gnjatic, 2010 [29] & ULK4 & $60 / 53$ & $7(2-16)$ & $100(93-100)$ & - & - \\
\hline Gnjatic, 2010 [29] & TMOD1 & $60 / 53$ & $7(2-16)$ & $98(90-100)$ & - & - \\
\hline Gnjatic, 2010 [29] & C8orf34 & $60 / 53$ & $7(2-16)$ & $100(93-100)$ & - & - \\
\hline Maacke, 2002 [22] & $\operatorname{Rad51}$ & $57 / 86$ & $7(2-17)$ & $100(96-100)$ & - & - \\
\hline Zhu, 2015 [50] & Brca1 & $41 / 135$ & $7(2-20)$ & $99(96-100)$ & - & $p<0.05$ \\
\hline Marxsen, 1994 [36] & p53 & $78 / 82$ & $6(2-14)$ & $98(91-100)$ & - & - \\
\hline Fyssas, 1997 [28] & thyroglobulin & $33 / 40$ & $6(1-20)$ & $98(87-100)$ & - & - \\
\hline Burfold, 2013 [25] & Muc1core3 glycopeptide & $35 / 247$ & $6(1-19)$ & $94(90-97)$ & - & $p>0.5$ \\
\hline Okada, 2005 [41] & SOX13 & $37 / 34$ & $5(1-19)$ & $100(90-100)$ & - & - \\
\hline Okada, 2005 [41] & MRPL12 & $37 / 34$ & $5(1-19)$ & $100(90-100)$ & - & - \\
\hline Okada, 2005 [41] & HMT1 & $37 / 34$ & $5(1-19)$ & $100(90-100)$ & - & - \\
\hline Okada, 2005 [41] & Tim44 & $37 / 34$ & $5(1-19)$ & $97(85-100)$ & - & - \\
\hline Okada, 2005 [41] & p53 & $37 / 34$ & $5(1-19)$ & $97(85-100)$ & - & - \\
\hline Gnjatic, 2010 [29] & FAM13A1 & $60 / 53$ & $5(1-14)$ & $100(93-100)$ & - & - \\
\hline Gnjatic, 2010 [29] & C17orf46 & $60 / 53$ & $5(1-14)$ & $100(93-100)$ & - & - \\
\hline Gnjatic, 2010 [29] & HERPUD1 & $60 / 53$ & $5(1-14)$ & $100(93-100)$ & - & - \\
\hline Gnjatic, 2010 [29] & AFG3L1 & $60 / 53$ & $5(1-14)$ & $100(93-100)$ & - & - \\
\hline Gnjatic, 2010 [29] & C4orf16 & $60 / 53$ & $5(1-14)$ & $100(93-100)$ & - & - \\
\hline Gnjatic, 2010 [29] & CD79B & $60 / 53$ & $5(1-14)$ & $100(93-100)$ & - & - \\
\hline Gnjatic, 2010 [29] & CRSP8 & $60 / 53$ & $5(1-14)$ & $100(93-100)$ & - & - \\
\hline Gnjatic, 2010 [29] & DNAJB1 & $60 / 53$ & $5(1-14)$ & $100(93-100)$ & - & - \\
\hline Gnjatic, 2010 [29] & NY-SAR-48 & $60 / 53$ & $5(1-14)$ & $100(93-100)$ & - & - \\
\hline Gnjatic, 2010 [29] & PPARG & $60 / 53$ & $5(1-14)$ & $100(93-100)$ & - & - \\
\hline Gnjatic, 2010 [29] & \begin{tabular}{|l} 
SHOC2 \\
\end{tabular} & $60 / 53$ & $5(1-14)$ & $96(87-100)$ & - & - \\
\hline Gnjatic, 2010 [29] & SMOX & $60 / 53$ & $5(1-14)$ & $100(93-100)$ & - & - \\
\hline Gnjatic, 2010 [29] & TMSB10 & $60 / 53$ & $5(1-14)$ & $100(93-100)$ & - & - \\
\hline Gnjatic, 2010 [29] & ZNF695 & $60 / 53$ & $5(1-14)$ & $100(93-100)$ & - & - \\
\hline Gnjatic, 2010 [29] & CRYBB2 & $60 / 53$ & $3(0-12)$ & $98(90-100)$ & - & - \\
\hline Gnjatic, 2010 [29] & ELAC1 & $60 / 53$ & $3(0-12)$ & $100(93-100)$ & - & - \\
\hline Gnjatic, 2010 [29] & HCFC1R1 & $60 / 53$ & $3(0-12)$ & $100(93-100)$ & - & - \\
\hline Burfold, 2013 [25] & Muc1STn glycopeptide & $35 / 247$ & $3(0-15)$ & 97 (94-99) & - & $p>0.5$ \\
\hline Okada, 2005 [41] & HAX1 & $37 / 34$ & $3(0-14)$ & $100(90-100)$ & - & - \\
\hline Okada, 2005 [41] & ZNF207 & $37 / 34$ & $3(0-14)$ & $100(90-100)$ & - & - \\
\hline Okada, 2005 [41] & RP-43L2 & $37 / 34$ & $3(0-14)$ & $100(90-100)$ & - & - \\
\hline $\mathrm{Li}, 2010[21]$ & Annexin A2 & $48 / 40$ & $2(0-11)$ & $100(91-100)$ & - & - \\
\hline Pekarikova, 2010 [23] & tTG & $55 / 56$ & $2(2-10)$ & $100(94-100)$ & - & - \\
\hline Johnston, 2009 [32] & Mesothelin IgG & $56 / 35$ & $0(0-10)$ & $97(85-100)$ & $>0.05$ & - \\
\hline $\mathrm{Li}, 2010[21]$ & HNRPA2 & $48 / 40$ & $0(0-7)$ & $100(91-100)$ & - & - \\
\hline Nakatsura, 2002 [39] & CLP peptide 57-65 IgG & $8 / 9$ & $0(0-37)$ & $100(66-100)$ & - & - \\
\hline Nakatsura, 2002 [39] & CLP peptide 57-65 IgE & $8 / 9$ & $0(0-37)$ & $89(52-100)$ & - & - \\
\hline Zhu, 2015 [50] & Parp1 & $41 / 135$ & $0(0-9)$ & $99(96-100)$ & - & - \\
\hline Zhu, 2015 [50] & Brca2 & $41 / 135$ & $0(0-9)$ & $99(96-100)$ & - & - \\
\hline
\end{tabular}




\begin{tabular}{|l|l|l|c|c|c|c|}
\hline Bracci, 2012 [24] & NR2E3 & $300 / 300$ & - & - & 0.56 & - \\
\hline Bracci, 2012 [24] & MAPK9 & $300 / 300$ & - & - & 0.59 & - \\
\hline Bracci, 2012 [24] & CTDSP1 & $300 / 300$ & - & - & 0.62 & - \\
\hline
\end{tabular}

Abbreviations: AFG3L1: AFG3 ATPase family gene 3-like 1; AL1A1: Retinal dehydrogenase 1; ARFIP2: ADP-ribosylation factor interacting protein 2; CEA: Carcinoembryonic antigen; CI: Confidence interval; CIB1: Calcium and integrin binding 1; COF1: Cofilin-1; CLP: Coactosin-like protein; CRYBB2: Crystallin beta B2; CTDSP1: Carboxy-terminal domain RNA polymerase II polypeptide A small phosphatase 1; DDX48: Dead-box protein48; DNAJB1: DnaJ (Hsp40) homolog subfamily B member 1; EGFR: epidermal growth factor receptor; EFTU: Elongation Factor Tu; ELAC1: ElaC homolog 1, ENOA: Alpha-enolase; HRY: hairy Drosophila-homolog; IDHC: Isocitrate dehydrogenase; FAM13A1: Family with sequence similarity 13 member A; GAS2: Growth arrest-specific 2; G6PD: Glucose-6-phosphate 1-dehydrogenase; HAX1: HS1 binding protein; HCFC1R1: Host cell factor C1 regulator 1; HERPUD1: Homocysteine-inducible endoplasmic reticulum stressinducible ubiquitin-like domain member 1; hPMS1: Homo sapiens postmeiotic segregation increased 1; hMSH2: Homo sapiens mutS homolog 2; HMT1: hnRNP methyltransferase; hnRNPL: Heterogeneous nuclear ribonucleoprotein L; HNRPA2: Heterogeneous nuclear ribonucleoprotein A2; IFITM3: Interferon-induced transmembrane protein 3; IMP1: IGF-II mRNA binding protein 1; K1C10: Keratin 10; Koc: KH-domain containing protein over expressed in cancer; LRRC49: Leucine rich repeat containing 49; MAPK: Mitogen-activated protein kinase; MDH1: Malate dehydrogenase; MIA: Melanoma-inhibitory activity; Muc1: Mucin 1; MRPL12: Mitochondrial ribosome protein L12; NR2E3: Nuclear receptor subfamily 2- group E- member 3; PARP1: poly (ADP-Ribose) Polymerase 1; PDC6I: Programmed Cell Death-6 Interacting protein; PGK1: Phosphoglyceratekinase 1; PNLIPRP2: Pancreas lipase-related protein 2; PPARG: Peroxisome proliferative activated receptor gamma; PSCA: Prostate stem cell antigen; PTPRA: Protein tyrosine phosphatase receptor type A; ROR2: Receptor tyrosine kinase-like orphan receptor 2; RPL29: 60S ribosomal protein L29, SHOC2: Soc-2 suppressor of clear homolog; SOX13: Sex determining region Y-box 13; TIM44: Translocase of inner mitochondrial membrane 44; TMSB10: Thymosin beta 10; tTG: Tissue transglutamase; SMOX: Spermine oxidase; TMOD1: Tropomodulin 1; TPIS: Triosephosphateisomerase 1; TAGL: Transgelin; ZNF207: Zinc finger protein 207; ZNF695: Zinc finger protein 695.

* $p$-value represents the statistical difference of positivity rate between cases and controls.

More advanced stages (III and IV) showed higher sensitivity for all the investigated autoantibodies. One study also assessed the tumor stage specific sensitivity of a combination of 6 different autoantibodies [35]. As can be seen from Supplementary Table S3 this led to enhanced sensitivity at all stages from $33 \%$ at stage I to $86 \%$ at stage IV [35]. Both studies also evaluated the tumor stage specific sensitivity for autoantibodies against p53 and again here some variability was seen between the studies.

Previous work has also carried out systematic reviews on the diagnostic performance of serum autoantibodies in colorectal cancer [51] and gastric

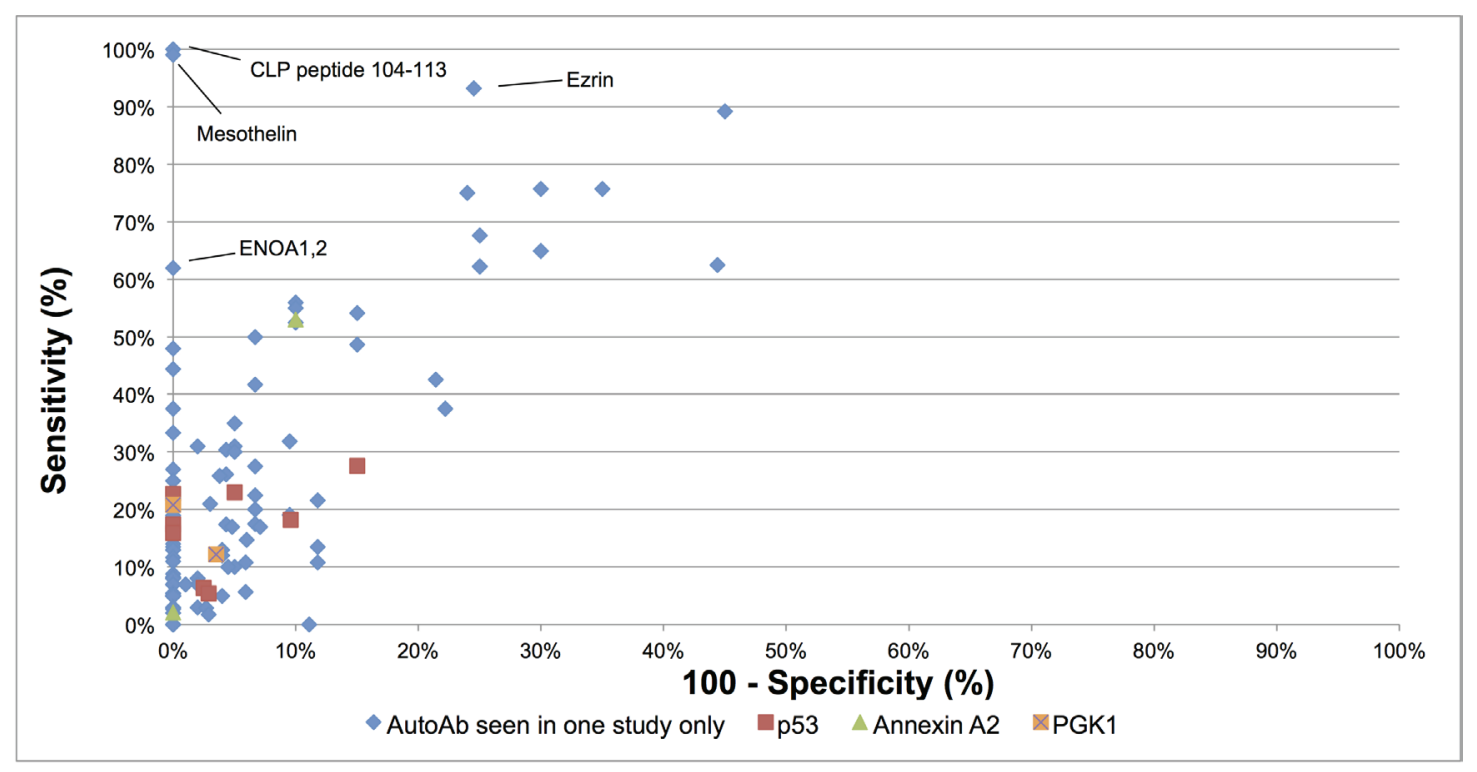

Figure 2: Graphical representation of sensitivity versus specificity of all analyzed autoantibodies. Sensitivity is plotted on the $y$-axis while on the $x$-axis the false positive rate is presented (100 - Specificity). Autoantibodies evaluated in one study only are labeled in blue. Autoantibodies evaluated in more than one study are labeled in red (p53), green (Annexin A2) and orange (PGK1). Autoantibodies that showed high sensitivity and specificity are labeled directly on the graph. Abbreviations: CLP: Coactosin-like protein; ENOA: Alphaenolase; PGK1: Phosphoglyceratekinase 1. 
Table 2: Autoantibodies examined in multiple studies

\begin{tabular}{|c|c|c|c|c|}
\hline \multirow{2}{*}{ Antigen } & References & No. of Studies & Sensitivity (\%) & Specificity (\%) \\
\hline p53 & {$[20,34-37,40,41,43]$} & 8 & $6-28$ & $85-90$ \\
\hline PGK1 & {$[21,42]$} & 2 & $12-21$ & $97-100$ \\
\hline Annexin A2 & {$[21,26]$} & 2 & $2-19$ & $90-100$ \\
\hline
\end{tabular}

Abbreviations: PGK1 Phosphoglyceratekinase 1

\section{Table 3: Diagnostic performance of marker combinations}

\begin{tabular}{|c|c|c|c|c|c|c|}
\hline $\begin{array}{l}\text { First author, } \\
\text { Year [Ref] }\end{array}$ & Marker Combination & $\begin{array}{c}\text { Cases }(\mathbf{N}) / \\
\text { Controls }(\mathbf{N})\end{array}$ & $\begin{array}{l}\text { Sensitivity \% } \\
\quad(95 \% \mathrm{CI})\end{array}$ & $\begin{array}{l}\text { Specificity \% } \\
\quad(95 \% \text { CI })\end{array}$ & $\mathbf{A U C}$ & $p$-value* \\
\hline $\begin{array}{l}\text { Capello, } 2013 \\
{[26]}\end{array}$ & $\begin{array}{l}\text { anti-Ezrin + anti- } \\
\text { ENOA1.2 + CA19,9 }\end{array}$ & $45 / 48$ & $100(92-100)$ & $92(80-98)$ & 0.96 & - \\
\hline $\begin{array}{l}\text { Tomaino, } 2011 \\
{[48]}\end{array}$ & $\begin{array}{l}\text { anti-ENOA1,2+ } \\
\text { CA19.9** }\end{array}$ & $37 / 63$ & $97(86-100)$ & $92(82-97)$ & 0.95 & $p=0.0001$ \\
\hline $\begin{array}{l}\text { Tomaino, } 2011 \\
{[48]}\end{array}$ & anti-ENOA1 + CA19.9*** & $61 / 63$ & $95(86-99)$ & $94(85-98)$ & 0.94 & $p=0.0001$ \\
\hline Tanaka, 2006 [46] & $\begin{array}{l}\text { anti-SART-109 + anti- } \\
\text { EGFR-479 + anti-Pap-112 } \\
+ \text { anti-EGFR-54 + anti- } \\
\text { CEA-425**** }\end{array}$ & $47 / 43$ & $83(69-92)$ & $88(75-96)$ & - & - \\
\hline Tanaka, 2007 [45] & $\begin{array}{l}\text { anti-PSCA peptide } 2-11+ \\
\text { anti-PSCA peptide } 86-95 \\
+ \text { anti-PSCA peptide } \\
109-118\end{array}$ & $40 / 60$ & $80(64-91)$ & $82(70-90)$ & - & - \\
\hline Syrigos, $1996[44]$ & $\begin{array}{l}\text { anti-Pancreatic islet b-cells } \\
+ \text { anti-Insulin }\end{array}$ & $36 / 21$ & $73(55-86)$ & $100(84-100)$ & - & $p<0.001$ \\
\hline Raedle, 1996 [43] & $\begin{array}{l}\text { anti-p53 + CA19.9 (Cutoff } \\
\text { 37U/ml) }\end{array}$ & $33 / 52$ & $73(54-87)$ & $64(50-76)$ & - & - \\
\hline $\mathrm{Li}, 2012[35]$ & $\begin{array}{l}\text { anti-p53 + anti-p16 + anti- } \\
\text { p62 + anti-Survivin + anti- } \\
\text { Koc + anti-IMP1 +CA19.9 }\end{array}$ & $23 / 23$ & $70(47-87)$ & - & - & - \\
\hline Tanaka, 2006 [46] & $\begin{array}{l}\text { anti-SART-109 + anti- } \\
\text { EGFR-479 + anti-Pap-112 } \\
+ \text { anti-EGFR-54 + anti- } \\
\text { CEA-425 ***** }\end{array}$ & $47 / 42$ & $64(49-77)$ & $71(55-84)$ & - & - \\
\hline $\mathrm{Li}, 2012[35]$ & $\begin{array}{l}\text { anti-p53 + anti-p16 + anti- } \\
\text { p62 + anti-survivin + anti- } \\
\text { Koc + anti-IMP1 }\end{array}$ & $23 / 23$ & $61(39-80)$ & $87(66-97)$ & - & $p<0.01$ \\
\hline Hong, 2004 [31] & $\begin{array}{l}\text { anti-Calreticulin isoform } \\
1+\text { anti-Calreticulin iso- } \\
\text { form } 2\end{array}$ & $36 / 15$ & $58(41-74)$ & $93(68-100)$ & - & - \\
\hline Raedle, 1996 [43] & $\begin{array}{l}\text { anti-p53 + CA19.9 (Cutoff } \\
\text { 100U/ml) }\end{array}$ & $33 / 52$ & $58(39-75)$ & $87(74-94)$ & - & - \\
\hline $\mathrm{Li}, 2012[35]$ & $\begin{array}{l}\text { anti-p53 + anti-p16 + } \\
\text { anti-p62 + anti-survivin }+ \\
\text { anti-Koc }\end{array}$ & $23 / 23$ & $52(31-73)$ & $91(72-99)$ & - & - \\
\hline Muller, 2006 [37] & anti-p53 + CA19-9 & $22 / 436$ & $50(28-72)$ & $100(99-100)$ & - & - \\
\hline
\end{tabular}




\begin{tabular}{|l|l|c|c|c|c|c|}
\hline Li, 2012 [35] & $\begin{array}{l}\text { anti-p53 + anti-p16+ anti- } \\
\text { p62 + anti-survivin }\end{array}$ & $23 / 23$ & $48(27-69)$ & $95(77-100)$ & - & - \\
\hline $\begin{array}{l}\text { Pekarikova, 2010 } \\
\text { [23] }\end{array}$ & $\begin{array}{l}\text { anti-Calreticulin IgA } \\
\text { anti-Calreticulin IgG }\end{array}$ & $55 / 56$ & $47(34-61)$ & $98(90-100)$ & - & - \\
\hline Li, 2010 [21] & $\begin{array}{l}\text { anti-PGK1 + anti-MPH1 } \\
\text { anti-ARFIP2 }\end{array}$ & $48 / 41$ & $46(31-61)$ & $100(91-100)$ & - & - \\
\hline Li, 2012 [35] & $\begin{array}{l}\text { anti-p53 + anti-p16+ anti- } \\
\text { p62 }\end{array}$ & $23 / 23$ & $39(20-61)$ & $96(78-100)$ & - & - \\
\hline Li, 2010 [21] & anti-PGK1 + anti-MPH1 & $48 / 40$ & $28(24-53)$ & $100(91-100)$ & - & - \\
\hline Li, 2012 [35] & anti-p53 + anti-p16 & $23 / 23$ & $35(16-57)$ & $96(78-100)$ & - & - \\
\hline Patwa, 2009 [42] & $\begin{array}{l}\text { anti-Histone H4 + anti- } \\
\text { PGK1 }\end{array}$ & $-/-$ & 33 & 94 & - & - \\
\hline Heller, 2010 [30] & $\begin{array}{l}\text { anti-MIA + anti-PNLIPR2 } \\
\text { + anti-IFITM2 }\end{array}$ & $34 / 20$ & $32(17-51)$ & $94(75-100)$ & - & $p=0.021$ \\
\hline Syrigos, 1996 [44] & $\begin{array}{l}\text { anti-Pancreatic islet b-cells } \\
\text { and anti-Insulin }\end{array}$ & $36 / 21$ & $31(16-48)$ & $100(84-100)$ & - & $p<0.001$ \\
\hline Ohshio, 2002 [40] & anti-p53 and CA19.9 & $82 / 21$ & $26(17-36)$ & - & - & - \\
\hline Fyssas, 1997 [28] & $\begin{array}{l}\text { anti-Microsomes + anti- } \\
\text { Thyroglobulin }\end{array}$ & $33 / 40$ & $25(11-42)$ & $95(76-100)$ & - & - \\
\hline Ohshio, 2002 [40] & anti-p53 and CEA & $82 / 21$ & $22(14-32)$ & - & - & - \\
\hline Fyssas, 1997 [28] & $\begin{array}{l}\text { anti-Microsomes and anti- } \\
\text { Thyroglobulin }\end{array}$ & $33 / 40$ & $3(0-16)$ & $98(87-100)$ & - & - \\
\hline Zhu, 2015 [50] & Parp1 and Brca1 & $41 / 135$ & $0(0-9)$ & $100(97-100)$ & - & - \\
\hline Zhu, 2015 [50] & Parp1 and Brca2 & $41 / 136$ & $0(0-9)$ & $100(97-100)$ & - & - \\
\hline Zhu, 2015 [50] & Brac1 and Brca2 & $41 / 137$ & $0(0-9)$ & $100(97-100)$ & - & - \\
\hline Zhu, 2015 [50] & $\begin{array}{l}\text { Parb1 and Brca1 and } \\
\text { Brca2 }\end{array}$ & $41 / 138$ & $0(0-9)$ & $100(97-100)$ & - & - \\
\hline
\end{tabular}

+ denotes and/or

${ }^{*} p$-value represents the difference of positivity rate between cases and controls.

$* *$ validation set

$* * *$ training set

$* * * *$ Discriminatory analysis

$* * * * *$ Cumulative analysis

Abbreviations: ARFIP2: ADP-ribosylation factor interacting protein 2; CEA: Carcinoembryonic antigen; CI: Confidence Interval;EGFR: epidermal growth factorreceptor;ENOA: Alpha-enolase;IFITM3:Interferon-induced transmembraneprotein 3; IMP1: IGF-IImRNAbinding protein 1;Koc:KH-domain containing proteinoverexpressed incancer; MIA: Melanoma-inhibitory activity PGK1: PARP1: poly (ADP-Ribose) Polymerase 1; Phosphoglyceratekinase 1; PNLIPRP2: Pancreas lipase-related protein 2; PSCA: Prostate stem cell antigen.

cancer [52]. Table 4 shows a comparison of the diagnostic performance of 9 different serum autoantibodies that have been analyzed in three gastrointestinal cancers. None of these markers seemed to be specific for one of the cancers only.

\section{DISCUSSION}

In this systematic literature review, we identified 31 studies on serum autoantibodies for the detection of pancreatic cancer that fit our inclusion criteria. The identified studies evaluated the diagnostic performance of 124 different serum autoantibodies. Overall, the diagnostic performance of individual autoantibody markers was quite limited, with $86 \%$ of markers showing less than $50 \%$ sensitivity. Of note, case numbers were mostly low internal and/or external validations were rarely implemented in these studies. Therefore, one needs to be cautious when interpreting the results.

Four autoantibodies (anti-CLP peptide 104-113, anti-Mesothelin, anti-Ezrin, anti-ENOA1,2) showed reasonable diagnostic performance (sensitivity greater than $60 \%$ and specificity greater than $80 \%$ ). However, one should also note that the performance of each these markers has only been reported in one study with mostly small numbers of cases and/or controls. The use 
Table 4: Comparison of diagnostic performance of autoantibodies in detecting three different cancers

\begin{tabular}{|c|c|c|c|c|}
\hline \multirow{2}{*}{ Antigen } & \multirow{2}{*}{ Test characteristic } & This Study & Chen et al. [51] & Werner et al. [52] \\
\hline & & Pancreatic cancer & Colorectal cancer & Gastric cancer \\
\hline \multirow{2}{*}{ p53 } & Sensitivity & $5 \%-28 \%$ & $9-46 \%$ & $8-32 \%$ \\
\hline & Specificity & $85 \%-90 \%$ & $90-100 \%$ & $95-100 \%$ \\
\hline \multirow{2}{*}{ Histone $\mathrm{H} 2 \mathrm{~B}$} & Sensitivity & $50 \%$ & $30 \%$ & $17 \%$ \\
\hline & Specificity & $93 \%$ & $92 \%$ & $93 \%$ \\
\hline \multirow{2}{*}{ p16 } & Sensitivity & $30 \%$ & $11 \%$ & $11 \%$ \\
\hline & Specificity & $96 \%$ & $99 \%$ & $99 \%$ \\
\hline \multirow{2}{*}{ p62 } & Sensitivity & $22 \%$ & $9-23 \%$ & $7-9 \%$ \\
\hline & Specificity & $100 \%$ & $97-99 \%$ & $98 \%$ \\
\hline \multirow{2}{*}{ Survivin } & Sensitivity & $17 \%$ & $4-56 \%$ & $8-10 \%$ \\
\hline & Specificity & $96 \%$ & $64-98 \%$ & $98 \%$ \\
\hline \multirow{2}{*}{ Koc } & Sensitivity & $22 \%$ & $9-15 \%$ & $16-19 \%$ \\
\hline & Specificity & $100 \%$ & $99-100 \%$ & $98 \%$ \\
\hline \multirow{2}{*}{ CEA } & Sensitivity & $17 \%$ & $9-64 \%$ & $52 \%$ \\
\hline & Specificity & $93 \%$ & $89-96 \%$ & $89 \%$ \\
\hline \multirow{2}{*}{ DDX48 } & Sensitivity & $33 \%$ & $10 \%$ & $7 \%$ \\
\hline & Specificity & $100 \%$ & $100 \%$ & $100 \%$ \\
\hline \multirow{2}{*}{ IMP1 } & Sensitivity & $26 \%$ & $13-22 \%$ & $17 \%$ \\
\hline & Specificity & $96 \%$ & $98-100 \%$ & $98 \%$ \\
\hline
\end{tabular}

Comparison of diagnostic performance of autoantibodies found in pancreatic cancer (this study), in colorectal cancer [52] and in gastric cancer [53]. Abbreviations: CEA: Carcinoembryonic antigen; DDX48: Dead-box protein48; Koc: KH-domain containing protein over expressed in cancer; IMP1: IGF-II mRNA binding protein.

of different autoantibody detection methods, different cutoff values chosen and patient samples representing different tumor stages might furthermore affect the generalizability of these findings. Therefore, validation of potential markers by independent studies is essential. Also, none of the markers have been looked at in a large-scale pancreatic cancer screening setting. Three of the four antibody recognized proteins mentioned above (Mesothelin, Ezrin, ENOA) are highly expressed in different cancers including pancreatic cancer [53-55]. Mesothelin, Ezrin and ENOA have also been linked to tumor metastasis and cancer progression. Not much has been reported on CLP (coactosin-like protein) and it is unclear if it is overexpressed or involved in pancreatic cancer. The molecular function of these proteins varies. Mesothelin is a GPI anchored cell surface protein that can promote cancer cell survival and proliferation [53], while Ezrin plays an important role in cellular processes like cell adhesion and migration and is linked to tumor metastasis [54]. Moreover high expression of Ezrin is associated with poor prognosis in different cancers including pancreatic cancer [ 56,57$]$. ENOA ( $\alpha$-enolase) is a metabolic enzyme that is important for glycolysis. It is also expressed on the cell surface where it acts as a receptor for plasminogen. As seen with Ezrin and Mesothelin, ENOA has been linked to cell migration and cancer metastasis [55]. CLP can bind actin and to 5-Lipoxygenase, a key enzyme involved in the biosynthesis of the inflammatory mediators Leukotrienes $[58,59]$, but less is known about its biological and molecular function.

Our analysis also indicates that the combination of multiple markers (either autoantibodies in combination with tumor associated markers or multiple autoantibodies) is likely the way forward as this improves sensitivity while not dramatically affecting specificity. The challenge remains to find out which combination of markers works best and this will require additional effort to sort out. The multi-marker regression model commonly used in this context may suffer from substantial over-optimism unless appropriate internal and/or external validations are carried out, which has often not been done in the past [60]. In addition, transparent reporting of a multivariable 
prediction model is essential for replication of study findings by other independent researchers. Future studies following the recently proposed TRIPOD statement by Collins and colleagues would strongly improve the overall validity of research findings [61].

What remains unclear is if autoantibodies are good markers for determining early stage pancreatic cancer, which is an important issue with pancreatic cancer treatment at the moment. Two studies reported sensitivity according to tumor stage and in both studies only advanced tumor stages showed reasonable sensitivity. However, given the small sample size one has to be careful what conclusions one can draw from this and clearly more work is needed to evaluate this. Finding biomarkers that can detect early stages of pancreatic cancer is a pressing concern and not a trivial one to address. One of the included studies [26] used as a starting point a spontaneous pancreatic cancer mouse mode to identify serum autoantibodies against TAA. The benefit of the mouse model is that sample size is not a limiting factor and that one has temporal control over when to collect the samples. Using this approach, serum autoantibodies to Ezrin were shown to develop early in the pancreatic cancer murine model and also in human patients with PDAC [26]. In this case, the mouse model worked as an effective screening tool and time will tell if this approach will aid in the discovery of early stage biomarkers for pancreatic cancer.

Many of the studies used a candidate approach when choosing what autoantibodies to analyze, while others used proteomic approaches without a priori defined targets. From the list of autoantibodies examined, it is fair to say that there is no good way of predicting which markers might work. Case in point is the tumor suppressor gene p53 that has been linked to many cancers and also pancreatic cancer and therefore would be a reasonable marker to investigate. 8 studies have examined anti-p53 in pancreatic cancer, but none of them reported high enough sensitivity to support that anti-p53 on its own could serve as a good marker for detecting pancreatic cancer. So the way forward is likely large scale and unbiased screens to identify the autoantibody signatures using well-defined tumor stage samples.

Lastly, all the included studies recruited participants in a clinical setting, i.e., cases were typically clinically diagnosed patients in hospitals, and convenient controls or healthy donors were used. Various key factors regarding specimens, such as blood sample collection, storage and handling, would introduce additional bias if not well controlled [62]. The choice of cutoff values may also make the comparison between studies difficult. Additionally, there is also some variability in what data and patient characteristics were reported in the published literature. Some studies did not report important information on age or male/female ratio. While others did not provide information on what type of control samples was used.
Agreement on what key factors need to be reported will help in comparing different studies and move the field forward. Also, while some studies used healthy participants as controls others used a mix of non-cancer patients, which also might make it difficult to draw comparisons between studies.

A particular challenge in diagnosing pancreatic cancer is the distinction of early pancreatic cancer from often, benign pancreatic diseases, which should receive particular attention in the selection of control groups in future studies. On the other hand, there is increasing evidence that autoantibodies against tumor-associated antigens may not be unique for specific types or locations of cancer. Possibly, autoantibodies signatures might therefore be best used as a screening tool to detect the presence of cancer in general to be followed by more specific diagnostic measures in case of a positive result.

To our knowledge, this is the first systematic literature review on serum autoantibodies as biomarkers for pancreatic cancer detection. There are some limitations that need to be considered when interpreting our review. Although we conducted a systematic search of relevant articles in two most widely-used databases and also adopted intensive cross-referencing, we cannot guarantee that all relevant studies have been identified. In addition, due to the larger heterogeneity in terms of study designs, detection methods and examined autoantibodies among included studies, a meta-analysis summarizing the diagnostic performance of markers was not meaningful.

To sum up, our review suggests that autoantibodies have the potential to be used as novel diagnostic markers for detecting pancreatic cancer possibly as part of a general cancer screening. However, current research in this area is still at a fairly early stage. More work is needed to identify promising autoantibody signatures and evaluate their diagnostic performance in detecting pancreatic cancer, especially at early stages. Given the limited diagnostic potential for single markers, multimarker combinations are needed to enhance the overall sensitivity. Future studies adopting more rigorous study designs and reporting well-adjusted diagnostic performance characteristics in a transparent manner would contribute greatly in this research area.

\section{MATERIALS AND METHODS}

The systematic literature review was carried out according to a predefined protocol. Reporting follows the PRISMA statement [63].

\section{Literature search}

A systematic literature search was carried out to identify studies that evaluated serum autoantibodies produced in pancreatic cancer patients and cancer free controls. PubMed (January 1, 1950 to April 27, 2015) 
and ISI Web of Knowledge (January 11945 to April 27, 2015) were searched for relevant articles that met our inclusion and exclusion criteria. The search was done using the following keyword combinations: [(pancreatic) and (cancer or neoplasm or carcinoma or adenoma or malignancy) and (autoantibodies or antibodies) and (detection or diagnosis or biomarker) and (serum or blood or plasma)] (Supplementary Table S1). Duplicated articles were removed. The initial screen was done based upon reading of the title and abstract. Articles that were not relevant to the topic were excluded. The second round of screening involved reading of the articles in full. In addition, we also identified a number of papers from crossreferencing (Figure 1).

\section{Eligibility criteria}

Only articles written in English were included in our review. Conference abstracts and reviews were excluded because of insufficiently reported information regarding diagnostic performance of autoantibody markers. We required that studies reported relevant information regarding diagnostic performance of autoantibody markers (e.g., sensitivity, specificity, area under the curve) for the detection of pancreatic cancer in humans as well as the numbers of cases and controls used in the studies. Studies not using cancer-free controls were further excluded.

\section{Data extraction}

Two reviewers (KD and $\mathrm{HC}$ ) independently read and retrieved data from the studies that met the above described inclusion and exclusion criteria. Any inconsistencies were discussed and resolved among the authors. We report the characteristics of study population (numbers of cases and controls, mean age and age range of study participants, male/female ratio and country where the study was performed), the health status of controls and the autoantibody detection method. The following diagnosis related indicators were extracted: overall and stage specific (if reported) sensitivity and specificity, area under the receiver operating characteristics curve (AUC). $95 \%$ confidence intervals $(95 \% \mathrm{CI})$ of sensitivities and specificities were calculated using medcalc software (https://www.medcalc.net/tests/diagnostic_test.php)

\section{ACKNOWLEDGMENTS AND FUNDING}

Financial support: HC's work is supported by China Scholarship Council (CSC).

\section{CONFLICTS OF INTEREST} interest.

\section{REFERENCES}

1. Siegel R, Ma J, Zou Z, Jemal A. Cancer statistics, 2014. CA Cancer J Clin. 2014; 64:9-29.

2. Yadav D, Lowenfels AB. The epidemiology of pancreatitis and pancreatic cancer. Gastroenterology. 2013; 144:1252-1261.

3. Ryan DP, Hong TS, Bardeesy N. Pancreatic adenocarcinoma. N Engl J Med. 2014; 371:1039-1049.

4. Solomon S, Das S, Brand R, Whitcomb DC. Inherited pancreatic cancer syndromes. Cancer J. 2012; 18:485-491.

5. Reznik R, Hendifar AE, Tuli R. Genetic determinants and potential therapeutic targets for pancreatic adenocarcinoma. Front Physiol. 2014; 5:87.

6. Canto MI, Harinck F, Hruban RH, Offerhaus GJ, Poley JW, Kamel I, Nio Y, Schulick RS, Bassi C, Kluijt I, Levy MJ, Chak A, Fockens P, et al. International Cancer of the Pancreas Screening (CAPS) Consortium summit on the management of patients with increased risk for familial pancreatic cancer. Gut. 2013; 62:339-347.

7. Ballehaninna UK, Chamberlain RS. Biomarkers for pancreatic cancer: promising new markers and options beyond CA 19-9. Tumour Biol. 2013; 34:3279-3292.

8. Zaenker P, Ziman MR. Serologic autoantibodies as diagnostic cancer biomarkers - a review. Cancer Epidemiol Biomarkers Prev. 2013; 22:2161-2181.

9. Desmetz C, Mange A, Maudelonde T, Solassol J. Autoantibody signatures: progress and perspectives for early cancer detection. J Cell Mol Med. 2011; 15:2013-2024.

10. Attallah AM, Abdel-Aziz MM, El-Sayed AM, Tabll AA. Detection of serum $\mathrm{p} 53$ protein in patients with different gastrointestinal cancers. Cancer Detect Prev. 2003; 27:127-131.

11. Kalkner KM, Ronnblom L, Karlsson Parra AK, Bengtsson M, Olsson Y, Oberg K. Antibodies against double-stranded DNA and development of polymyositis during treatment with interferon. QJM. 1998; 91:393-399.

12. Muro S, Miyake Y, Kato H, Tsutsumi K, Yamamoto K. Serum anti-60S ribosomal protein L29 antibody as a novel prognostic marker for unresectable pancreatic cancer. Digestion. 2015; 91:164-173.

13. Angelopoulou K, Diamandis EP. Autoantibodies against the P53 Tumor-Suppressor Gene-Product Quantified in CancerPatient Serum with Time-Resolved Immunofluorometry. Cancer Journal. 1993; 6:315-321.

14. Hamanaka Y, Suehiro Y, Fukui M, Shikichi K, Imai K, Hinoda Y. Circulating anti-MUC1 IgG antibodies as a favorable prognostic factor for pancreatic cancer. Int $\mathbf{J}$ Cancer. 2003; 103:97-100.

15. Kusama M, Kusama K, Hayashi S, Sunouchi T, Chu L, Moro I. Natural antibody against Thomsen-Friedenreich antigen in sera of patients with carcinomas and infectious diseases. J Nihon Univ Sch Dent. 1993; 35:241-243. 
16. Patwa TH, Wang Y, Simeone DM, Lubman DM. Enhanced detection of autoantibodies on protein microarrays using a modified protein digestion technique. Journal of Proteome Research. 2008; 7:2553-2561.

17. Suzuki H, Graziano DF, McKolanis J, Finn OJ. T celldependent antibody responses against aberrantly expressed cyclin B1 protein in patients with cancer and premalignant disease. Clin Cancer Res. 2005; 11:1521-1526.

18. Talar-Wojnarowska R, Gasiorowska A, Olakowski M, Dranka-Bojarowska D, Lampe P, Smigielski J, Kujawiak M, Grzegorczyk J, Malecka-Panas E. Utility of serum IgG, IgG4 and carbonic anhydrase II antibodies in distinguishing autoimmune pancreatitis from pancreatic cancer and chronic pancreatitis. Advances in Medical Sciences. 2014; 59:288-292.

19. Kotera Y, Fontenot JD, Pecher G, Metzgar RS, Finn OJ. Humoral immunity against a tandem repeat epitope of human mucin MUC-1 in sera from breast, pancreatic, and colon cancer patients. Cancer Res. 1994; 54:2856-2860.

20. Gansauge S, Gansauge F, Negri G, Galle P, Muller J, Nussler AK, Poch B, Beger HG. The role of anti-p53autoantibodies in pancreatic disorders. Int $\mathrm{J}$ Pancreatol. 1996; 19:171-178.

21. LiC, Kim HY, Vuong H, Patwa T, Pal M, Brand RE, Simeone DM, Lubman DM. The identification of auto-antibodies in pancreatic cancer patient sera using a naturally fractionated Panc-1 cell line. Cancer Biomark. 2010; 7:25-37.

22. Maacke H, Hundertmark C, Miska S, Voss M, Kalthoff H, Sturzbecher HW. Autoantibodies in sera of pancreatic cancer patients identify recombination factor Rad51 as a tumour-associated antigen. J Cancer Res Clin Oncol. 2002; 128:219-222.

23. Pekarikova A, Sanchez D, Palova-Jelinkova L, Simsova M, Benes Z, Hoffmanova I, Drastich P, Janatkova I, Mothes T, Tlaskalova-Hogenova H, Tuckova L. Calreticulin is a B cell molecular target in some gastrointestinal malignancies. Clin Exp Immunol. 2010; 160:215-222.

24. Bracci PM, Zhou M, Young S, Wiemels J. Serum autoantibodies to pancreatic cancer antigens as biomarkers of pancreatic cancer in a San Francisco Bay Area case-control study. Cancer. 2012; 118:5384-5394.

25. Burford B, Gentry-Maharaj A, Graham R, Allen D, Pedersen JW, Nudelman AS, Blixt O, Fourkala EO, Bueti D, Dawnay A, Ford J, Desai R, David L, et al. Autoantibodies to MUC1 glycopeptides cannot be used as a screening assay for early detection of breast, ovarian, lung or pancreatic cancer. British Journal of Cancer. 2013; 108:2045-2055.

26. Capello M, Cappello P, Linty FC, Chiarle R, Sperduti I, Novarino A, Salacone P, Mandili G, Naccarati A, Sacerdote C, Beghelli S, Bersani S, Barbi S, et al. Autoantibodies to Ezrin are an early sign of pancreatic cancer in humans and in genetically engineered mouse models. J Hematol Oncol. 2013; 6:67.

27. Falco A, Rosati A, Festa M, Basile A, De Marco M, d'Avenia M, Pascale M, Dal Piaz F, Tavano F, Di Mola FF, di Sebastiano P, Berloco PB, Nudo F, et al. BAG3 is a novel serum biomarker for pancreatic adenocarcinomas. Am J Gastroenterol. 2013; 108:1178-1180.

28. Fyssas I, Syrigos KN, Konstantoulakis MM, Tsibloulis V, Manouras A, Peveretos P, Golematis BC. Thyroid autoantibodies and thyroid function in patients with pancreatic adenocarcinoma. Acta Oncol. 1997; 36:65-68.

29. Gnjatic S, Ritter E, Buechler MW, Giese NA, Brors B, Frei C, Murray A, Halama N, Zoernig I, Chen Y-T, Andrews C, Ritter G, Old LJ, et al. Seromic profiling of ovarian and pancreatic cancer. Proceedings of the National Academy of Sciences of the United States of America. 2010; 107:5088-5093.

30. Heller A, Zornig I, Muller T, Giorgadze K, Frei C, Giese T, Bergmann F, Schmidt J, Werner J, Buchler MW, Jaeger D, Giese NA. Immunogenicity of SEREX-identified antigens and disease outcome in pancreatic cancer. Cancer Immunol Immunother. 2010; 59:1389-1400.

31. Hong SH, Misek DE, Wang H, Puravs E, Giordano TJ, Greenson JK, Brenner DE, Simeone DA, Logsdon CD, Logsdon D, Hanash SM. An autoantibody-mediated immune response to calreticulin isoforms in pancreatic cancer. Cancer Research. 2004; 64:5504-5510.

32. Johnston FM, Tan MCB, Tan BR, Jr., Porembka MR, Brunt EM, Linehan DC, Simon PO, Jr., Plambeck-Suess S, Eberlein TJ, Hellstrom KE, Hellstrom I, Hawkins WG, Goedegebuure P. Circulating Mesothelin Protein and Cellular Antimesothelin Immunity in Patients with Pancreatic Cancer. Clinical Cancer Research. 2009; 15:6511-6518.

33. Kamei M, Kato M, Mochizuki K, Kuroda K, Sato S, Hashizume S, Yasumoto K, Murakami H, Nomoto K. Serodiagnosis of Cancers by ELISA of Antihistone H2B Antibody. Biotherapy. 1992; 4:17-22.

34. Laurent-Puig P, Lubin R, Semhoun-Ducloux S, Pelletier G, Fourre C, Ducreux M, Briantais MJ, Buffet C, Soussi T. Antibodies against $\mathrm{p} 53$ protein in serum of patients with benign or malignant pancreatic and biliary diseases. Gut. 1995; 36:455-458.

35. Li J, Wang LJ, Ying X, Han SX, Bai E, Zhang Y, Zhu Q. Immunodiagnostic Value of Combined Detection of Autoantibodies to Tumor-associated Antigens as Biomarkers in Pancreatic Cancer. Scandinavian Journal of Immunology. 2012; 75:342-349.

36. Marxsen J, Schmiegel W, Roder C, Harder R, Juhl H, Henne-Bruns D, Kremer B, Kalthoff H. Detection of the anti-p53 antibody response in malignant and benign pancreatic disease. Br J Cancer. 1994; 70:1031-1034.

37. Mueller M, Meyer M, Schilling T, Ulsperger E, Lehnert T, Zentgraf H, Stremmel W, Volkmann M, Galle PR. Testing for anti-p53 antibodies increases the diagnostic sensitivity of conventional tumor markers. International Journal of Oncology. 2006; 29:973-980.

38. Nagayoshi Y, Nakamura M, Matsuoka K, Ohtsuka T, Mori Y, Kono H, Aso T, Ideno N, Takahata S, Ryo A, Takeda H, Ito T, 
Oda Y, et al. Profiling of autoantibodies in sera of pancreatic cancer patients. Ann Surg Oncol. 2014; 21:S459-465.

39. Nakatsura T, Senju S, Ito M, Nishimura Y, Itoh K. Cellular and humoral immune responses to a human pancreatic cancer antigen, coactosin-like protein, originally defined by the SEREX method. Eur J Immunol. 2002; 32:826-836.

40. Ohshio G, Suwa H, Imamura M. Clinical implication of anti-p53 antibodies and p53-protein in pancreatic disease. Int J Gastrointest Cancer. 2002; 31:129-135.

41. Okada T, Noji S, Goto Y, Iwata T, Fujita T, Okada T, Matsuzaki Y, Kuwana M, Hirakata M, Horii A, Matsuno S, Sunamura M, Kawakami Y. Immune responses to DNA mismatch repair enzymes hMSH2 and hPMS1 in patients with pancreatic cancer, dermatomyositis and polymyositis. International Journal of Cancer. 2005; 116:925-933.

42. Patwa TH, Li C, Poisson LM, Kim H-Y, Pal M, Ghosh D, Simeone DM, Lubman DM. The identification of phosphoglycerate kinase-1 and histone $\mathrm{H} 4$ autoantibodies in pancreatic cancer patient serum using a natural protein microarray. Electrophoresis. 2009; 30:2215-2226.

43. Raedle J, Oremek G, Welker M, Roth WK, Caspary WF, Zeuzem S. p53 autoantibodies in patients with pancreatitis and pancreatic carcinoma. Pancreas. 1996; 13:241-246.

44. Syrigos KN, Konstantoulakis MM, Fyssas I, Katsilambros N, Golematis BC. Autoantibodies against insulin and beta-islet cells in pancreatic adenocarcinoma: a possible explanation for diabetes mellitus. Int J Cancer. 1996; 66:624-626.

45. Tanaka M, Komatsu N, Terakawa N, Yanagimoto Y, Oka M, Sasada T, Mine T, Gouhara S, Shichijo S, Okuda S, Itoh K. Increased levels of IgG antibodies against peptides of the prostate stem cell antigen in the plasma of pancreatic cancer patients. Oncol Rep. 2007; 18:161-166.

46. Tanaka M, Komatsu N, Yanagimoto Y, Oka M, Shichijo S, Okuda S, Itoh K. Development of a new diagnostic tool for pancreatic cancer: simultaneous measurement of antibodies against peptides recognized by cytotoxic $\mathrm{T}$ lymphocytes. Kurume Med J. 2006; 53:63-70.

47. Tomaino B, Cappello P, Capello M, Fredolini C, Ponzetto A, Novarino A, Ciuffreda L, Bertetto O, De Angelis C, Gaia E, Salacone P, Milella M, Nistico P, et al. Autoantibody signature in human ductal pancreatic adenocarcinoma. J Proteome Res. 2007; 6:4025-4031.

48. Tomaino B, Cappello P, Capello M, Fredolini C, Sperduti I, Migliorini P, Salacone P, Novarino A, Giacobino A, Ciuffreda L, Alessio M, Nistico P, Scarpa A, et al. Circulating autoantibodies to phosphorylated alpha-enolase are a hallmark of pancreatic cancer. J Proteome Res. 2011; 10:105-112.

49. Xia Q, Kong XT, Zhang GA, Hou XJ, Qiang H, Zhong RQ. Proteomics-based identification of DEAD-box protein 48 as a novel autoantigen, a prospective serum marker for pancreatic cancer. Biochemical and Biophysical Research Communications. 2005; 330:526-532.
50. Zhu Q, Han SX, Zhou CY, Cai MJ, Dai LP, Zhang JY. Autoimmune response to PARP and BRCA1/BRCA2 in cancer. Oncotarget. 2015; 6:11575-11584. doi: 10.18632/ oncotarget.3428.

51. Chen H, Werner S, Tao S, Zornig I, Brenner H. Blood autoantibodies against tumor-associated antigens as biomarkers in early detection of colorectal cancer. Cancer Lett. 2014; 346:178-187.

52. Werner S, Chen H, Tao S, Brenner H. Systematic review: serum autoantibodies in the early detection of gastric cancer. Int J Cancer. 2015; 136:2243-2252.

53. Pastan I, Hassan R. Discovery of mesothelin and exploiting it as a target for immunotherapy. Cancer Res. 2014; 74:2907-2912.

54. Clucas J, Valderrama F. ERM proteins in cancer progression. J Cell Sci. 2014; 127:267-275.

55. Capello M, Ferri-Borgogno S, Cappello P, Novelli F. alphaEnolase: a promising therapeutic and diagnostic tumor target. FEBS J. 2011; 278:1064-1074.

56. Piao J, Liu S, Xu Y, Wang C, Lin Z, Qin Y, Liu S. Ezrin protein overexpression predicts the poor prognosis of pancreatic ductal adenocarcinomas. Exp Mol Pathol. 2015; 98:1-6.

57. Li J, Wei K, Yu H, Jin D, Wang G, Yu B. Prognostic Value of Ezrin in Various Cancers: A Systematic Review and Updated Meta-analysis. Sci Rep. 2015; 5:17903.

58. Provost P, Doucet J, Stock A, Gerisch G, Samuelsson B, Radmark O. Coactosin-like protein, a human F-actinbinding protein: critical role of lysine-75. Biochem J. 2001; 359:255-263.

59. Basavarajappa D, Wan M, Lukic A, Steinhilber D, Samuelsson B, Radmark O. Roles of coactosin-like protein (CLP) and 5-lipoxygenase-activating protein (FLAP) in cellular leukotriene biosynthesis. Proc Natl Acad Sci U S A. 2014; 111:11371-11376.

60. Siontis GC, Tzoulaki I, Castaldi PJ, Ioannidis JP. External validation of new risk prediction models is infrequent and reveals worse prognostic discrimination. Journal of clinical epidemiology. 2015; 68:25-34.

61. Collins GS, Reitsma JB, Altman DG, Moons KG. Transparent reporting of a multivariable prediction model for individual prognosis or diagnosis (TRIPOD): the TRIPOD Statement. BMC medicine. 2015; 13:1.

62. Ransohoff DF, Gourlay ML. Sources of bias in specimens for research about molecular markers for cancer. Journal of Clinical Oncology. 2010; 28:698-704.

63. Moher D, Liberati A, Tetzlaff J, Altman DG, The PRISMA Group. Preferred Reporting Items for Systematic Reviews and Meta-Analyses: The PRISMA Statement. PLoS Medicine. 2009; 6:e1000097. 